There were also three papers on activation analysis. Activation by thermal neutrons is now well established. Because several active nuclides are usually present after irradiation it is necessary either to perform radiochemical separations or - in favourable cases-to use multichannel gamma spectrometry to measure active species. Mr D. Mapper described improvements in separation methods and the advance made possible by solid state detectors and the use of data processing and computer techniques.

There are some notable omissions from the list of elements which can be determined by thermal neutron activation. Dr C. A. Baker illustrated this with reference to three important impurities in metallurgy - carbon, nitrogen and oxygen. Irradiation with $30 \mathrm{MeV}$ gamma rays produces radioactive neutron deficient isotopes-carbon-11, for example-which can be used to determine these elements at the p.p.m. level in samples weighing a few hundred milligrams.

Large and expensive equipment is required for activation by thermal neutrons and high energy gamma rays. Dr T. B. Pierce described the recent development of sealed-tube valve type neutron generators using the deuterium-tritium reaction. This makes possible a relatively inexpensive, compact system for the production of fast neutrons and is particularly suitable for the analysis of oxygen, aluminium, silicon, vanadium, chromium and titanium in intact metallurgical samples.

\section{Inhibitory Synapses}

\section{from our Neurophysiology Correspondent}

THE structure of the neuromuscular junction and of synapses in the central nervous system have been intensively studied by electron microscopists; it is fair to say that the physical basis for the storage and release of transmitter substances is well understood. Indeed, within the great diversity of synapses studied there is a "remarkable uniformity in the structures which are believed to be essentially concerned in their functional operation" (Eccles). Usually a presynaptio fibre expands to form a synaptic knob; within this, close to the presynaptic membrane, are large numbers of round vesicles between 300 and $600 \AA$ in diameter. It is universally accepted (although most of the evidence is circumstantial) that the synaptic vesicles contain "packets" of transmitter molecules.

Recently Uchizono has suggested that there might be an anatomical basis for the recognition of excitatory and inhibitory synapses (Nature, 20\%, 642; 1965). Several authors have described synapses containing flattened, rather small, vesicles; Uchizono made the hypothesis that these were characteristic of inhibitory synapses. In the current issue of Experimental Brain Research $(4,97 ; 1967)$ he describes an attempt to correlate vesicular shape with synaptic function in the cerebellar cortex of cats. Eccles and his colleagues have used the cerebellar cortex as a model system because its structure is well known and conceptually simple. As they have been able to identify many of the gross anatomical features with specific physiological properties and in particular have shown which elements exert excitatory and which inhibitory effects, the cerebellum is ideal for Uchizono's purpose. The cerebellar cortex receives excitation through two sets of inputs-the mossy fibres and the climbing fibres. Mossy fibres synapse with granule cells in the "mole- cular" layer of the cortex: these give rise to axons which run vertically to the granular layer and there bifurcate, forming the parallel fibres which run along the long axes of the folds in the cerebellar cortex. As the course of the parallel fibres is so characteristic, their synapses are easily identifiable. They are known to be excitatory, and Uchizono shows that they contain spheroidal vesicles. The climbing fibres, which synapse with the dendrites of cerebellar Purkinje cells, are also known to be excitatory; their synapses contain spheroidal vesicles. On the other hand, the synapses of the basket cells, also easily identifiable, are known to be inhibitory: these contain ellipsoidal vesicles. Other synapses containing flattened or ellipsoidal vesicles are tentatively identified as belonging either to stellate or basket cells, both inhibitory.

So far, then, the evidence from the cerebellar cortex supports Uchizono's hypothesis. Several objections, however, have been made to it: Andersen and others have suggested that identification of the origin of a terminal axon can only be certain when supported by degeneration studies. However, it seems reasonable to accept Uchizono's claim of unequivocal identification on the grounds of our knowledge of cerebellar structure. It has also been suggested that ellipsoidal vesicles are fixation artefacts. While it is known that this can be so, several of Uchizono's electron micrographs show neighbouring structures containing round and ellipsoidal vesicles. A more serious possibility is that the same transmitter might be shown to have opposite effects in different synapses-as acetylcholine has on heart muscle and skeletal muscle-for presumably one type of transmitter inhabits only one sort of vesicle. Unequivocal support for Uchizono's hypothesis can only come from identification of the transmitter molecules in the two types of vesicle. Even so, it is at the moment more successful than the previous attempts to link synaptic structure with function.

\section{Algal Toxins \\ from our Microbiology Correspondent}

IN comparison with bacterial and fungal toxins, algal toxins have had little attention. This is somewhat surprising because algae cause the well known fresh water and saline toxic blooms and "poisonous red tides", which present hazards to the health of humans, domestic animals and marine life. Indeed, some algal toxins are among the most potent known; that of Gonyaulax catenella, for example, has a lethality approaching that of botulin. Plankton-feeding shellfish have been implicated as vectors of algal toxins, but for the intoxication of other aquatic animals large scale toxin release induced by autolytic, bacteriolytic or algal virus action appears to be a pre-requisite. So far very few algal toxins have been characterized chemically: that of $G$. catenella is a substituted purine while Microcystis aeruginosa produces a cyclie polypeptide toxin containing D-serine. The mode of action of these substances involves the blocking of axonal and muscle conduction by disturbing sodium or calcium conductance. Apart from studying the production of algal toxins from the viewpoint of animal intoxication, it would also seem profitable to use these substances as tools in the investigation of excitable membranes and also to evaluate their possible antibiotic activities. 